\title{
Exploring Social Interaction with Everyday Object based on Perceptual Crossing
}

\author{
Siti Aisyah binti Anas, Shi Qiu, Matthias Rauterberg, Jun Hu \\ Eindhoven University of Technology, \\ Department of Industrial Design, \\ $5600 \mathrm{MB}$, Eindhoven, The Netherlands \\ \{s.a.b.anas, sqiu, g.w.m.rauterberg, j.hu\}@tue.nl
}

\begin{abstract}
Eye gaze plays an essential role in social interaction which influences our perception of others. It is most likely that we can perceive the existence of another intentional subject through the act of cathing one another's eyes. Based on the notion of perceptual crossing, we aim to establish a meaningful social interaction that emerges out of the perceptual crossing between a person and an everyday object by exploiting the gazing behavior of the person as the input modality for the system. We investigated in literature the experiments that adopt the perceptual crossing as their foundation, lessons learned from literature were used as input for a concept to create meaningful social interaction. We used an eye-tracker to measure gaze behavior that allows the participant to interact with the object by using their eyes through active exploration. It creates a situation where both of them mutually becoming aware of each other's existence. Further, we discuss the motivation for this research, present a preliminary experiment that influences our decision and our directions for future work.
\end{abstract}

\section{Author Keywords}

Perceptual crossing; eye tracking; gaze sensitive object; human-object interaction; social interaction.

\section{ACM Classification Keywords}

H.5.m. Information Interfaces and Presentation (e.g. HCI): Miscellaneous.

\section{INTRODUCTION}

The growing maturity of sensor technology allows engineers to transform everyday object and make it smart enough for effortless interaction with users. Human perceptual sensing capabilities seem becoming limited and in many cases underappreciated as the technology grows. It seems that

Permission to make digital or hard copies of all or part of this work for personal or classroom use is granted without fee provided that copies are not made or distributed for profit or commercial advantage and that copies bear this notice and the full citation on the first page. Copyrights for components of this work owned by others than ACM must be honored. Abstracting with credit is permitted. To copy otherwise, or republish, to post on servers or to redistribute to lists, requires prior specific permission and/or a fee. Request permissions from Permissions@acm.org. HAI '16, October 04-07, 2016, Biopolis, Singapore

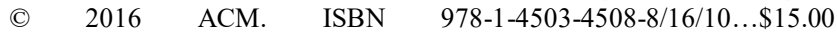
DOI: http://dx.doi.org/10.1145/2974804.2974810 people are looking for perfection without even realizing that they are missing something that they normally use to experience the world - their capabilities to interact in this world. Taking coffee machine as an example, we simply push a button, and its all done for us. Of course, technology helps us in so many ways, but through this technology, we experience our everyday object without meaningful, and expressive interaction.

We are capable of experiencing and interacting with the world in very different ways because of our built-in multitude of senses - sight, hearing, taste, smell and touch. We have these abilities that seem to fade away as the technology evolves through the years. This world is full of things that are right in front of us, but we did not realize their existence unless we expect to see them. We interact and experience things around us effortlessly without being aware of it. Taking the experience of looking into the mirror and seeing the image reflected back. We perceive ourselves as the image starts to mimic our behavior. The image looks back, and our gaze crosses each other. However, most of us do not realize the existence of the mirror, the object which helps us to make sure that we are still the same person we were yesterday. What if the mirror was aware that we are looking at it and we get the feeling that the mirror looks back at us? How could the mirror respond and what could it do to look back at us? The psychoanalyst, Jacques Lacan believe that the act of seeing is a reciprocal process [5]. According to him, each object has a certain presence that makes him feel of being looks back. "I see and I can see that I am seen, so each time I see, I also see myself being seen." However, not everyone can feel the same as Lacan sees things. Indeed, we tend to less appreciate the act of seeing things in front of us. The presence of objects that make our live complete is nothing more than just an object. The question remains, though, on how can we make the object and the person aware of each other's existence. Under what situation can we experience meaningful social interaction with everyday object? What if we change the way we look at things and the things we look at change in the sense that it reacts to our gaze? Can we create situations when we look at something and it "looks" back at us? 


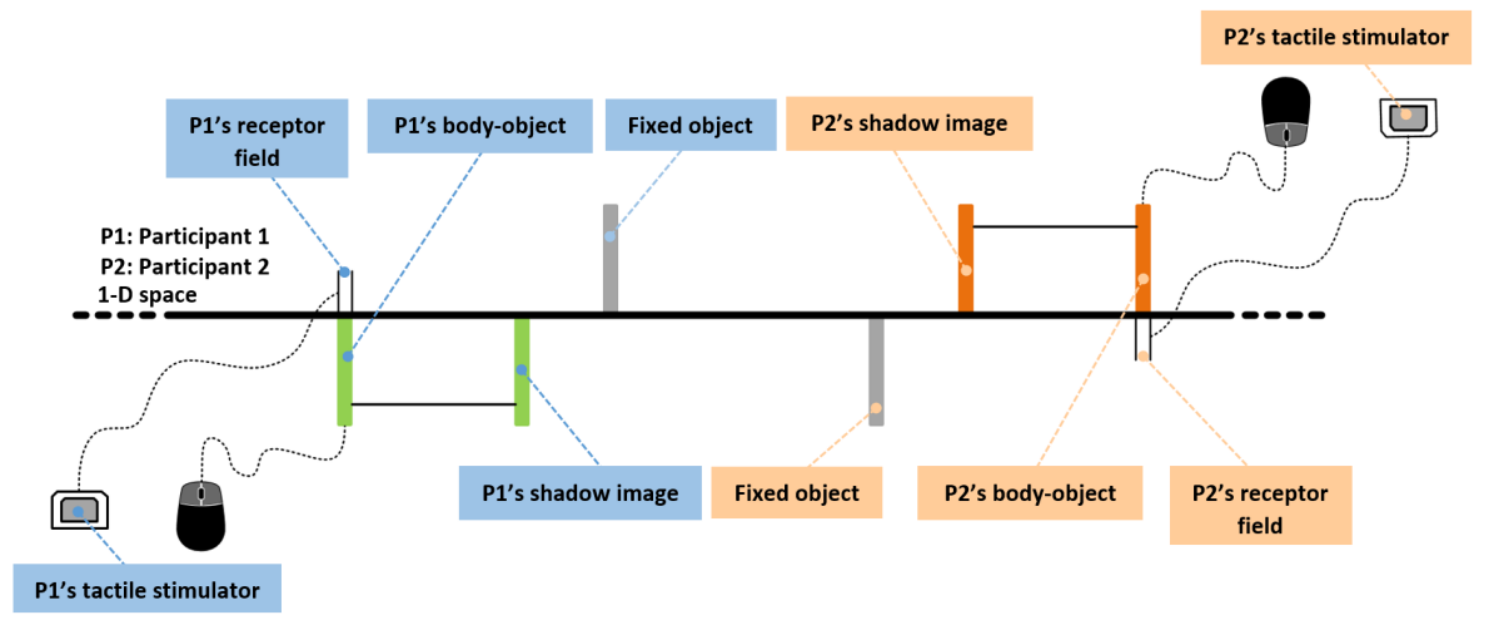

Figure 1. Virtual environment of Auvrey et. al's perceptual crossing paradigm [1].

\section{RELATED WORK}

\section{Perceptual Crossing Paradigm}

Auvrey et al.'s [1] perceptual crossing paradigm (PCP) is a well-known paradigm for studying real-time interaction and has been used by many researchers in different areas to investigate factors involved in perceiving each other's existence. In their experiment [1], pairs of participants in separate rooms explored a one-dimensional virtual space using a computer mouse and received tactile stimuli on the index finger of the other hand if they encountered something in space. There were three objects that participants could encounter: the partner's body-object, a fixed object and the shadow image which movement was identical to their partner's body-object. The only difference between the body-object and the shadow image was that the former was responsive to the perceptual crossing (both participants would receive tactile stimuli when they encountered each other). The task was to click the mouse button when the participants believed that they perceived the presence of their partner (see Figure 1). The results indicated that participants clicked more often when they encountered the partner's body-object. The participants' ability to distinguish all three objects resulted from active exploration and shared perceptual activity that influenced their behavior during realtime interaction and not because they consciously recognized the differences between three of the objects. When they encountered each other, both of them received tactile stimulation. They would reverse the direction of their bodyobject that caused them to performed the same oscillatory behavior. The co-dependence of the two participants to coordinate their behavior influenced them to create a stable interaction.

Froese et al. [6] later continued to investigate the dynamics of interaction process based on PCP by using Evolutionary Robotics simulation modeling. There were two agents in the simulation, and they needed to locate each other's presence. Once both agents successfully established perceptual crossing, they needed to maintain the interaction until the end of the trial. For this task, both agents seemed to depend on the duration of the stimulation to differentiate whether they encountered their partner, the static object or their partner's shadow image. That has led to a conclusion that the agents might use individual strategy to carry out the task and not because they mutually perceived each other. Based on this result, the team created another task where they switched the receptor field of both agents. The agents should be able to complete the task if they mutually depended on each other during interaction process even though they could not rely on their receptor field at the individual level. As predicted, the agents successfully established perceptual crossing that self-organizes out of the interaction process. It is arguable that both agents perceived one another because they were actively forming the interaction based on their individual efforts to stay in contact rather than relying on each other during interaction process. The creation of the last task was to prove the validity of this argument. Instead of mutually responding toward each other, agents were required to remained in contact with their partner's shadow image. If both agents depend on their individual strategy to complete this task, stayed in contact with the shadow image would be easy. It turned out that both agents could not meet the task requirement successfully. Unstable interaction occurred when they were trying to interact with the shadow image, hence prevented the establishment of perceptual crossing from occurring out of the interaction process. However, during exploration, when both agents found each other, they somehow sustained the interaction even though the task required them to stay in contact with their partner's shadow image. It showed that the interaction process itself shaped the behavior of the agents rather than depending on the individual strategy to complete the task.

Lenay et al. [12] studied the emergence patterns produced by the participants during the interaction process in a twodimensional virtual space. The task of this experiment was still the same as Auvrey's PCP where participants needed to clicked when they encountered their partner's body-object. 
Through active exploration, participants employed several criteria based on their trajectory in space and time. The act of anticipation formed when sufficient regularity occurred during interaction with a fixed object. Participants expected the stimulation to be consistent when they perceived a fixed object. The act of surprised to differentiate between a fixed object with their partner's body-object and the shadow image (the other). When participants encountered a fixed object, the stimulation should be regular (the act of anticipation) as the object did not move in space. However, when they failed to establish these regularities (the act of surprised), it can be concluded that they were in contact with the other. The other's existence were unpredictable but could also retain its presence if the other was their partner's body-object. Logically, these criteria employed by the participants were based on the encountered of two intentionalities during the perceptual crossing. The participants did not need to recognize each other before they established perceptual crossing. It is because they mutually responded during perceptual crossing that led them to recognize each other.

Lizuka et al. [13] investigated whether participants could differentiate the interaction to be live or recorded. Based on Auvrey et al.'s PCP but without the static object and the shadow image in the virtual space, participants would encounter two kinds of body-objects. One was the bodyobject controlled by their partner. The other was the recorded body-object from the previous trial. The participants faced difficulties at the beginning but after several trials, they developed a turn-taking behavior. During the live interaction, Participant 1 (P1) oscillated at the body-object of Participant 2 (P2) while P2 stayed at one place and identified P1's oscillatory behavior. When P1 stopped moving and oscillating, P2 would repeat the same behavior as he/she recalled from P1. The establishment of turn-taking behavior by $\mathrm{P} 1$ and $\mathrm{P} 2$ made this strategy useful in determining whether the interaction was live or not. Lizuka et al. also extended this experiment by investigating whether noncommunicative behavior could become communicative when both participants encountered each other. In this experiment, pairs of participants needed to decide whether they confronted with an identical or different shape (sharp,\# or square, $\square$ ) displayed on the monitor. After several trials, both participants learned to take turns and communicated by exchanging oscillation patterned to represent each shape that they confronted during the trial. If they saw a sharp, the oscillation patterned become more frequent and fast. If they saw a square, the movement of the oscillation become slower. Lizuka et al.'s experiment showed that during the interaction process, participants were capable of developing turn-taking behavior to communicate with each other through non-verbal interaction.

Deckers et al. [4] proposed six design notions to establish perceptual crossing between a person and an artefact. Focus the Senses, Active Behavior Object, Subtleness, Reaction to External Event, Detecting Active Behavior Subject, Reflecting Contextual Noise, and Course of Perception in
Time were implemented by them in designing an artefact which they addressed as the perception pillar plus $(P E P+)$. $P E P+$ was a square pillar embedded with eight ultrasonic sensors, and 17 static matrices of LEDs mounted on top surface of the pillar. An experiment conducted between $\mathrm{PEP}+$ and the participant concluded that most of the participants managed to achieve shared perception with the artefact that was one of the vital element during perceptual crossing. The possibility of experiencing reciprocal perception improved the participant's feeling of beeing involved during interaction with the artefact within the environment.

\section{Lesson Learned from Perceptual Crossing}

Social interaction can emerge out of the perceptual crossing. Even though participants do not have any conscious recognition to interact with each other, but through active exploration in real-time, they can perceive one another and mutually become aware of their partner's existence. This process is crucial for them to understand the social interaction [2]. Social interaction often lead to a social understanding based on the collective properties gain during the interaction process. Traditionally, social understanding is built upon how we predict and interpret other people's behavior or action. However, the concept of PCP is to concentrate on the interaction process rather than focusing on how individual figures out the others, but taking interaction as a medium to recognize and to engage with one another [9]. An example was during the encounter with the body-object and the shadow image in the aforementioned experiments. The experimenter's intuition was the difficulties that participants might face differentiating the body-object with the shadow image as both of the object move in the same manner. However, during the interaction process, when participants encounter the shadow image, they established unstable interaction which leads them to avoid the shadow image and proceed to explore the space until they perceive a stable interaction [1].

One can also observe in the aforementioned experiments several patterns adopted by participants during the interaction process. The act of surprise and anticipation when they encountered the body-object, fixed object and the shadow image that helped them to recognize which is which [12]. They learned the turn-taking behavior to distinguish whether the interaction was live or not [13]. They managed to use this turn-taking behavior as a strategy to communicate with each other by controlling their oscillation patterned [13].

During the simulation, it seemed that both agents might come out with an individual strategy to differentiate the objects they encountered [6]. However, when the experimenter switched the receptor field of both agents and changed the task to which they needed to stay in contact with the shadow image of the other agent, both agents somehow managed to establish perceptual crossing but failed to interact with the shadow image. Both agents preferred to stay in contact with 
each other even though the task told them no to do so. The interaction process influenced both agent's behavior to establish perceptual crossing.

All of these behaviors, patterns and criteria were established during active exploration that self-organized out of the interaction process without any explicit interpretation of the other interactor. It did not concern on the individual's ability to act in order to complete the task, but the shared perceptual activity was the most important part that influences their behavior to act during the interaction. They learned to appreciate each other existence in the very limited resources. Even though it required some time, eventually they managed to perceive each other's presence. Variations of results gained from the aforementioned experiments provoke inspiration to design interactive devices that can establish meaningful social interaction with a person during the interaction process itself.

\section{MOTIVATION}

This research is motivated to appreciate human capabilities and to create opportunities to experience social interaction that emerges out of the perceptual crossing with an everyday object. We aim to design an interactive everyday object that is sensitive to the perceptual crossing by depending on the user's gaze behavior as the input modality for the system. Giddens et al. [7] addresses that social interactions is a process of acting and reacting toward people around us, and it can be in any forms of verbal and nonverbal communication. Since visual modality is dominant for most individuals and as the matter of fact, our eyes serve as the focal points of our body, our gaze behavior is an essential type of nonverbal communication. We use our eyes to perceive others and to signal our intentions [8]. Hence, we decided to make use the role of gaze in developing social interaction with an object. Through active exploration, we wanted to establish a situation where the person and the object could coordinate their behavior so that both of them would mutually become aware of each other existence.

\section{PROTOTYPE}

\section{Mechanical Actuating}

To design an object that can perceive while being perceived, it needs to possess distinctive characteristics that allow it to be engaged in live dyadic interactions. We decided to use a coffee cup as our first everyday object that we commonly use in our daily lives. Figure 2 shows the exploded view drawing of the coffee cup. Table 1 outlines the explanation for each part.

The purpose of this design is to allow the cup the ability to react during interaction with the user. We believe that by adding the coffee cup with elements of dynamic behavior, the user can experience social interaction with it, and the cup's movement is triggered only when they look at it. To control the movement of the cup, we used gears as part of the working mechanism.

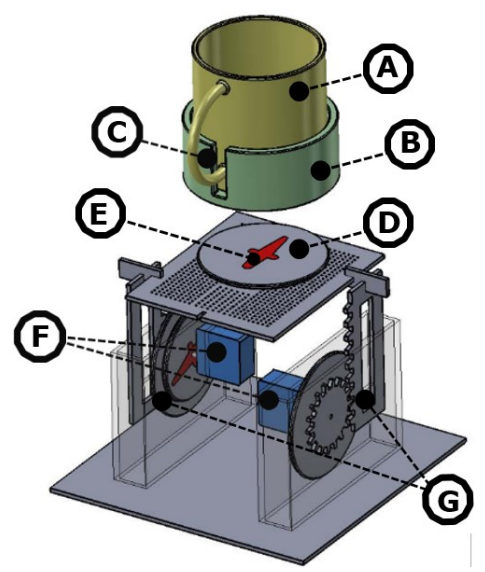

Figure 2. Exploded view drawing of the coffee cup.

\begin{tabular}{c|c} 
Part & $\begin{array}{c}\text { Explanation } \\
\text { The coffee cup }\end{array}$ \\
A & $\begin{array}{c}\text { The cup's holder to hold the cup } \\
\text { securely on top of the base Below } \\
\text { The slot to grip the cup's handle } \\
\text { The base to control the cup's rotation } \\
\text { (rotate left or right) } \\
\text { C }\end{array}$ \\
D & $\begin{array}{c}\text { Servo horn } \\
\text { E }\end{array}$ \\
F & $\begin{array}{c}\text { Combination of rack and pinion to } \\
\text { allow the cup to move vertically (up } \\
\text { or down) }\end{array}$ \\
G & $\begin{array}{c}\text { Servo motors to control the } \\
\text { movement of the cup }\end{array}$
\end{tabular}

Table 1. The explanation for each part of the design.

A pinion is a typical round gear, and a rack is a straight bar with teeth that allow it to engage with the pinion's gear teeth. When the pinion rotates, it causes the rack to move corresponding to the pinion, thereby enable the cup to move up if the pinion rotates clockwise, or move down if the pinion rotates counterclockwise (see Figure 3).

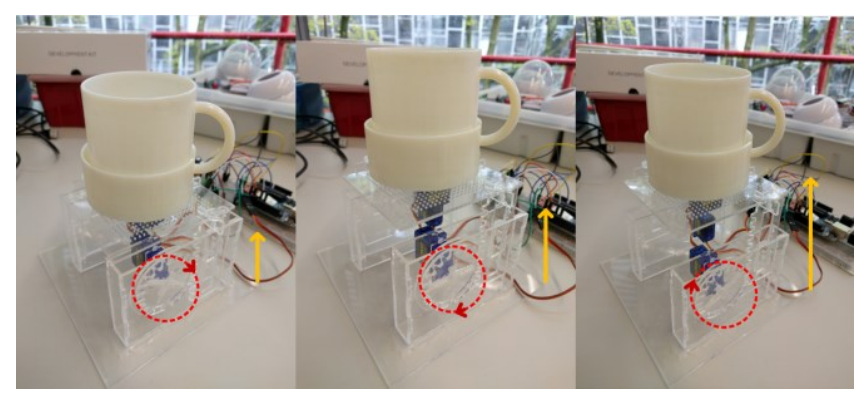

Figure 3. The coffee cup moves up when the pinion rotates clockwise.

The cup's holder (B) and the base (D) are securely fixed together with plastic screws. This base is attached to a servo horn where one servo motor control the rotation of the coffee cup to a specified position (see Figure 4). We used acrylic plastic as the material for the mechanical parts and Adobe 
Illustrator to generate the design for laser cutting. The coffee cup (A) and the cup's holder (B) were modeled using Rhino3D and fabricated using Ultimaker's 3D printer.

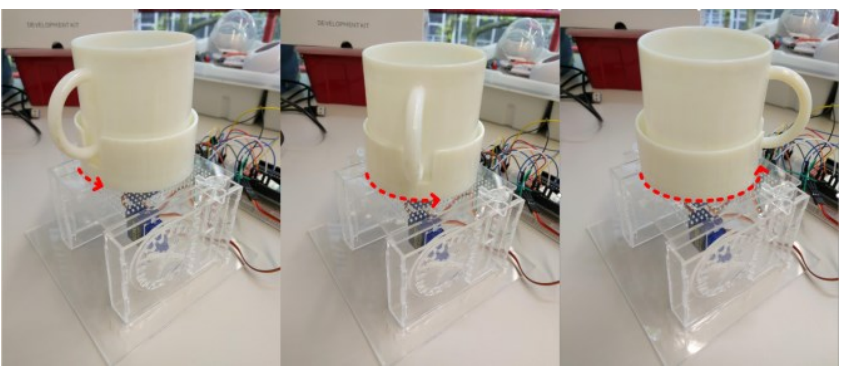

Figure 4. The coffee cup rotates to a specified position (from left to right).

\section{Sensing: Eye Tracking}

To track the gaze behavior in real-time, we need a device that can measure the participant's eye positions and movements. The Eye Tribe Tracker is an affordable eye tracker that can detect and determine the point of gaze defined by a pair of $(\mathrm{x}, \mathrm{y})$ coordinates with an average efficiency of $0.5^{\circ}$ to $1.0^{\circ}$ of visual angle and it connects to a USB 3.0 port on a laptop. It comes with software that allows client applications to access the underlying tracker's server to obtain a real-time stream of gaze data in raw and smoothed forms. To create a system that is responsive toward the user's eye gaze, we developed a Java program to calculate and reveal the location of the gaze point on the screen. A graphical user interface (GUI) with 15 targeted area is created to detect the point of interest where the user fixates in real-time. Figure 5 depicts the interface of the program. When the user fixates on one of the targeted areas, the system will activate the correspond circle (large red circle) to indicate that the user is now looking at that point (example: Point 3, Point 7). The small red dot indicates the user's current point of gaze. We assigned each targeted area with a command. Once the targeted area is activated, this command is sent out to an Arduino over a wireless Bluetooth connection.

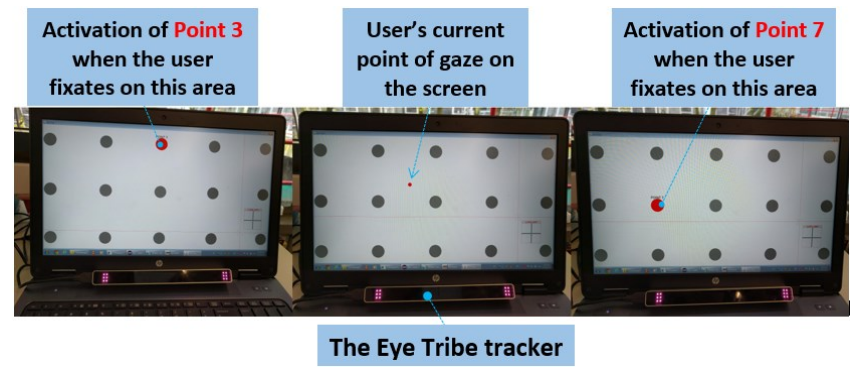

Figure 5. The interface of the system.

\section{Processing and Behavior}

To enable the coffee cup to interact and respond towards the user's eye gaze, the position of the cup is carefully predetermined, and must be within the eye tracker's tracking area. From Figure 6, it can be seen that the position of the coffee cup is equal to Point 7 from the GUI. The eye tracker detects the user's eye gaze, and if the user's gaze point correspond to the position of the coffee cup, a command is sent out via Bluetooth adapter from the laptop to a Bluetooth module connected to an Arduino.

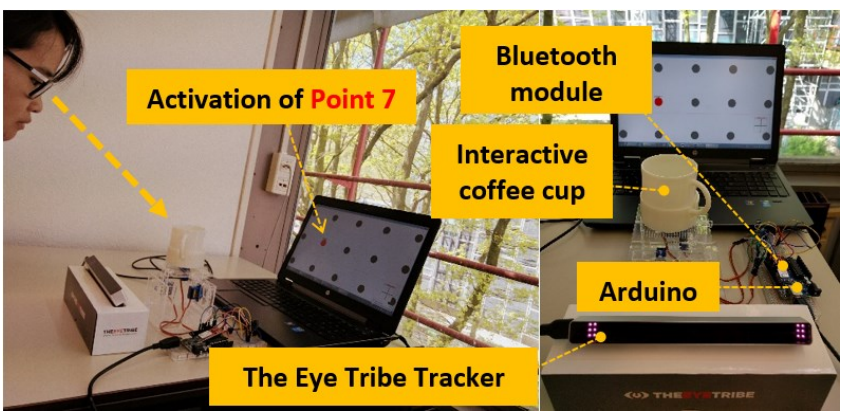

Figure 6. A user is fixating at the interactive coffee cup.

This microcontroller process the signal received from the module, and it generates behavior to the interactive coffee cup. There are four modes of behavior that the interactive coffee cup could display when the user is looking at it. For this experiment, Arduino will decide which mode displayed to the user in a random manner. If the user is not looking at the cup, it will go back to its initial state. Table 2 shown the type of behavioral patterns of the interactive coffee cup. These patterns depend on the degrees of rotation that the servo motors will rotate during interaction with the user. All of these patterns were created to be use in future experiment where the user will engage more with the coffee cup. Breathing happen when the coffee is still in the cup and the user ignore the cup (by not looking at it) for a while. Shivering occur when the temperature of the coffee in the cup drop to a certain value. Playing result when the user fixates at the cup for a certain time but does not pick it up to drink the coffee. Dancing happens when the cup is empty, and the user is looking at the cup.

\begin{tabular}{|l|l|l|}
\hline Type & Behavioral patterns & Mode \\
\hline & Initial \\
State
\end{tabular}




Type

Table 2. Coffee cup's behavioral patterns.

\section{EXPERIMENT}

\section{Participants}

15 participants, nine men, and six women, between the ages of 26 to 38 participated in the experiment. Seven of them is a Ph.D. student, five are currently not working, and three of them work as an engineer from various field.

\section{Setup}

We want to investigate whether participants can perceive the behavior of the coffee cup that will only react when they look at it. We decided to place a normal coffee cup beside the interactive coffee cup. We expect the participant to compare both cups and explore the environment by shifting their gaze during the experiment. Can the participant and the cup perceive each other behavior during his/her active exploration? Figure 7 illustrate the experimental setup. The placement of the interactive coffee cup was very crucial and must correspond with the system's targeted area. The position of the participant was also important and must be paralleled to the eye tracker's tracking area. It is necessary to center aligned the tracker and adjusted it towards the participant's face for the maximum trackability. When the participant gazes at the setup, the eye tracker extracted the gaze coordination and compared it with the pre-determined position of the interactive coffee cup. If matched, a command is sent via Bluetooth and Arduino will display the behavioral patterns of the interactive coffee cup to the participant in a random manner.

Since eye gaze is not a very typical input modality used for interaction in a real environment and mostly confined to the digital or virtual world, we want to investigate whether participants realize that their eye gaze is being exploited by the system that allows them to interact with the coffee cup. If the participant look away from the interactive coffee cup, it will go back to its initial state (type 0). If the participant fixates at the interactive coffee cup, it will react (random mode) to indicate "I see you looking at me. Hence, I will respond."

Normally, we rely heavily on sight to guide our movements that lead to appropriate actions [15]. However, for this experiment, we want the participants to appreciate the joy of experiencing social interaction with an object by simply looking at it and without the needs for them to act during the ongoing experiment.

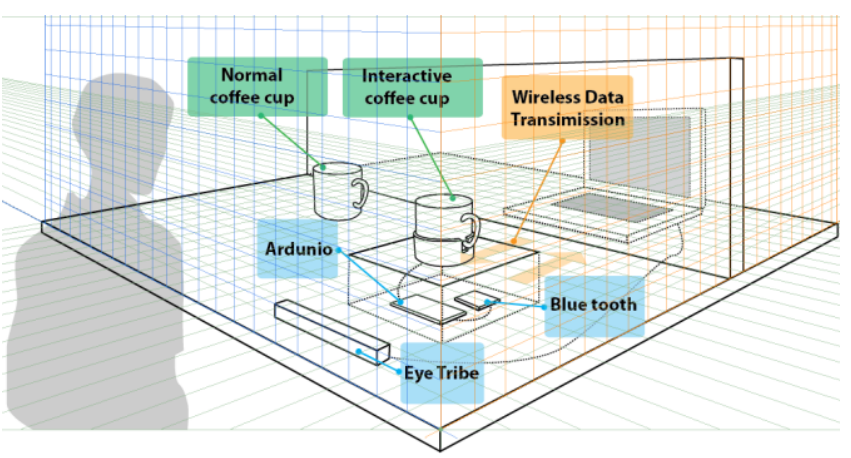

Figure 7. Overview of the setup.

\section{Procedures}

The participants were told to sat in front of a desk where the normal and the interactive coffee cup were placed. The instructions were rather simple. We instructed the participants to observe both coffee cup, and at the end of the experiment, they filled out a questionnaire. After that, we asked two open-ended questions related to the participant's perception regarding both cups. If the open-ended question leaves the participants confused, we explained to them the concept and motivation behind this research and asked them to participate in the experiment once again. Participants followed the same instructions and filled out the same questionnaire for the second time.

\section{Questionnaire}

We decided to measure the participants experienced with the interactive coffee cup by using the User Experience Questionnaire (UEQ) [10]. The questionnaire consists of 6 scales with 26 items. Attractiveness indicates the overall impression of the product. Perspicuity illustrates the difficulty of participants to get familiar and learn how to use the product. Efficiency is the ability of participants to solve the task without unnecessary effort. Dependability indicates if participants feel in control during the interaction process. Stimulation shows the excitement and motivation of participants to use the product. Novelty indicates whether the product is innovative, creative and able to catch the participant's interest [10]. Two open-ended questions regarding the participant's experienced during observation of both coffee cups were constructed and delivered to them. The questions were; what is the difference between these two cups and did the participant realize that his/her gaze is making the cup react. Verbal and non-verbal responses gathered from the participants were also recorded for future references and were used to complement the findings from the UEQ.

\section{RESULTS}

We categorized the findings from the UEQ into two, the before and the after condition. The first experiment was the before condition where the participants were instructed to 
observed the coffee cups without knowing the motivation behind the experiment. The second experiment was the after condition where the participants were asked to participate in the experiment once again after we explained to them the objectives of this research. Figure 6 shows the scores for all scales based on findings from the UEQ.

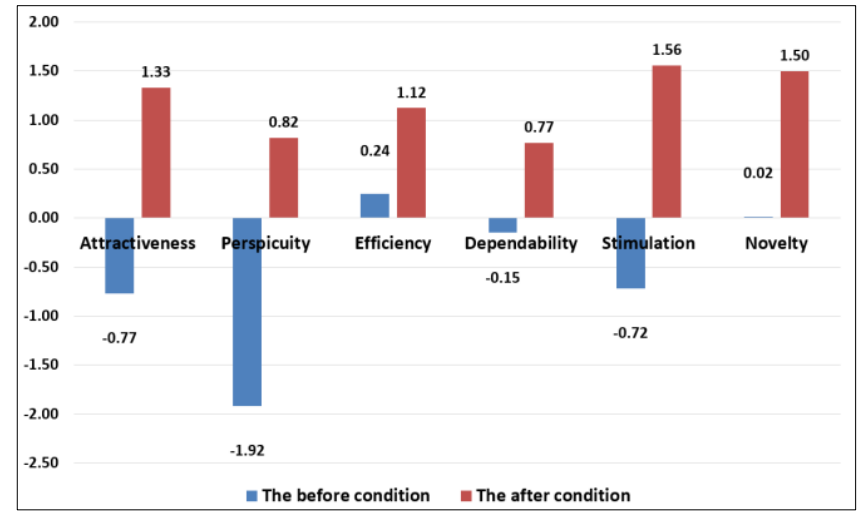

Figure 6. Graph of the six UEQ scales

From the graph, the overall scores indicated clearly the difference for each scales between the before and the after condition. The participant's responses to the open-ended questions may explain these findings. 13 out of 15 participants faced the difficulties to explain the differences between the regular coffee cup with the interactive one other than the former was a static object, and the later was embed with mechanical behaviors that allowed the cup to show random movements. The same participants also did not realize that their gaze influenced the interactive cup to react. The cup perceived participants' gaze, but participants did not perceive the cup's reaction to their gaze at the same time. Since the task was to observe on both objects without any further instructions or explanation, most of the participants seemed to be confused on why the interactive cup was suddenly showing some behaviors and why at some time it stopped interacting. The participant's gaze tended to focus more on the interactive cup rather than to compare and to observe their gaze with the other normal coffee cup. They felt that the cup was interacting on its own that attracted them to fixate on the interactive cup. They were also busy trying to interpret the behavior shown by the coffee cup which was rather new to them and if the cup's behavior was related to their action or influenced by the environment. However, after the second attempt, the graph show major improvements on the scores indicating positive feedback from participants. They started to explore both objects actively and realized that their gaze indeed influenced the cup's behavior. It was after being told the motivation behind this research that they felt the existence of the cup rather that just simply an object that could demonstrate random movement. Four of the participants found the interactive cup playful and very responsive. Six of the participants felt that the cup was trying to communicate with them, but they could not interpret it as the behavior was very random and unrecognizable. Others thought that the cup was a toy. Participant 3 said: "I cannot believe that we can interact with the cup just by depending on our eye gaze. It is fascinating and new." Participant 5 said: "It is weird to see the cup with this kind of behavior, but I like the idea behind this research." Participant 10 said: "This research can attract children to observe their surroundings rather than spending more time with the touchscreen. Like my kids!". Furthermore, all of the participants did not realize the function of the eye tracker in front of them. They thought that it was just part of the interactive design.

\section{DISCUSSION}

The observation during experiments, findings from the UEQ and feedback gathered from the participants gave us some hints regarding on why the user did not perceive the behavior of the coffee cup while the coffee cup perceive the user's gaze behavior and the future if this research. These can be summarized as follows:

\section{- Active exploration is crucial in social interaction}

The accuracy of the interactive cup that can react once it detects the presence of someone's gaze does not give much impact on the participant's feeling of mutually becoming aware of each other's existence. The interaction process needs to be improved in this context. In PCP, active exploration during the interaction process is crucial to make both subjects feel the presence of each other. We cannot simply depend on the accuracy of the input to produce the output or participants will face difficulties to understand the environment. We need to consider on how to build the relationship through active exploration which can lead to the discovery of each other presence. The establishment of the relationship can be developed when the object and the subject understand and recognize each other's behavior.

\section{- Behavior of the interactive object}

It is not necessary to design an interactive object that has unique features to perform perceptual crossing. "Unique" here means that overloading too many new features to an existing everyday object will divert our primary purpose of creating social interaction with the object. Perhaps, the use of properties already built in the object can be modified to create unique characteristics. An analog clock, for example, its mechanical behavior of controlling the moving hands can be altered as part of its unique features that can be used to perform perceptual crossing. Otherwise, participants will be preoccupied constructing internal interpretation of the object's behaviors that is very new to them rather than realizing the interaction process with the object.

\section{- The identity of the system}

For the system to have its identity, it needs to consider stages of data processing cycle and not to rely on producing an output whenever the system detect an input. This method is crucial in PCP as the system needs to actively explore and become familiar with the environment first in order to recognize the presence of the others. Possibly, the system should self-organize its behavior during the interaction 
process rather than depend on a set of predefined rules. The system should consider to analyze the characteristics of the gaze behavior, interpret the necessary gaze information and exploit the gaze behavior to produce relevant output [14]. However, the system must not consume too much time for its behavior to emerge, or participants might lose interest to interact as there is no feedback coming from the system in time.

\section{- The eye tracking metrics}

To develop the system's identity during the interaction process, it needs to understand the participant's gaze behavior since it is the only reliable input modality that the system can use. Gaze behaviors can reveal our point of interest. The eye tribe tracker enables us to collect and measure gaze data in real-time. It can detect the points of gaze of the participants. The time spent observing the point and traces of fixation patterns from a specific point to another reflects how participants scan the environment. These eye tracking metrics are useful to draw a better picture in obtaining direct feedback from participants while they interact with the object [3], which can contribute to the development of the system's behavior.

\section{CONCLUSION AND FUTURE WORK}

From our preliminary experiment, even though the task was rather simple, we gain insights that can guide us in creating meaningful social interaction between a person and an everyday object. The accuracy of the system to produce the output is important when both the subject and the object perceive each other rather than to depend on the accuracy of the sensor to detect an input and straightaway produce the output without considering the interaction process. The object should identify and adopt the behavioral patterns of the subject through the learning process and coordinate its behavior once it recognizes the subject. Furthermore, to design an interactive everyday object, we need to investigate and fully utilize the characteristic of the existing object. The object's behavior needs to be relevant to the object itself. This way, we can reduce internal representations constructed by the subject and allow the subject to appreciate the social interaction with the object that can emerge out of the perceptual crossing during the interaction process. Unless we want to attract the subject's attention, then a "unique" design should be applicable.

We view the work describe in this paper as a starting point for our research. We intend to create a system that can detect human's gaze behavioral patterns which will influence the system's ability to respond during perceptual crossing. We also intend to develop a complete framework that can enable a person to experience social interaction with everyday objects by depending on his/her gazing behavior.

\section{ACKNOWLEDGMENTS}

We thank our colleagues at the Design Intelligence research group who give suggestions and ideas for this project. This research received funding from the Ministry of Higher
Education, Malaysia and Universiti Teknikal Malaysia Melaka (UTeM).

\section{REFERENCES}

[1] Auvray, M., Lenay, C. and Stewart, J. 2009. Perceptual interactions in minimalist virtual environment. New Ideas Psychol. 27, (2009), 79-97.

[2] Auvray, M. and Rohde, M. 2012. Perceptual crossing: the simplest online paradigm. Frontiers in Human Neuroscience. 6, (2012), 181-194.

[3] Bergstrom, J.R. and Schall, A. 2014. Eye Tracking in User Experience Design. Morgan Kaufmann.

[4] Deckers, E., Lévy, P., Wensveen, S., Ahn, R. and Overbeeke, K. 2013. Designing for perceptual crossing: applying and evaluating design notions. International Journal of Design. 6, (2013), 41-55.

[5] Elkins, J. 1997. The Object Stares Back. On the Nature of Sensing. Simon and Schuster, Inc.

[6] Froese, T. and Di Paolo, E.A. 2009. Modeling social interactions as perceptual crossing: an investigation into dynamics of the interaction process. Connection Science. 22, (2009), 43-68.

[7] Giddens, A., Duneier, M., Appelbaum, R.P. and Carr, D. 2013. Introduction to Sociology. W. W. Norton \& Company.

[8] Gobel, M.S., Heejung, S.K. and Richardson, D.C. 2015. The dual function of social gaze. International Journal of Cognitive Science. 136, (2015), 359-364.

[9] De Jaegher, H. 2009. Social understanding through direct perception? Yes, by interacting. Consciousness and Cognition. 18, (2009), 535-542.

[10] Laugwitz, B., Held, T. and Schrepp, M. 2008. Construction and evaluation of a User Experience Questionnaire. USAB. 5298, (2008), 63-76.

[11] Lenay, C. and Stewart, J. 2012. Minimalist approach to perceptual interactions. Frontiers in Human Neuroscience. 6, (2012), 98-115.

[12] Lenay, C., Stewart, J., Rohde, M. and Ali Amar, A. 2011. You never fail to surprise me: the hallmark of the Other. Experimental study and simulations of perceptual crossing: Interaction Studies. 12(3), (2011), 373-396.

[13] Lizuka, H., Marocco, D., Ando, H. and Maeda, T. 2012. Turn-taking supports humanlikeness and communication in perceptual crossing experiments. Virtual Reality Short Papers and Posters (VRW) IEEE. (2012), 1-4.

[14] Nakano, Y.I., Conati, C. and Bader, T. 2013. Eye Gaze in Intelligent User Interfaces. Gaze-based Analyses, Models and Applications. Springer.

[15] Noe, A. 2006. Action in Perception (Representation and Mind series). A Bradford Book. 